\title{
LOCAL MODEL NETWORKS FOR MODELLING AND PREDICTIVE CONTROL OF NONLINEAR SYSTEMS
}

\author{
Jakub Novak, Petr Chalupa and Vladimir Bobal \\ Tomas Bata University, Faculty of Applied Informatics \\ Department of Process Control \\ Nad Stranemi 4511, Zlin 76005, Czech Republic \\ E-mail: jnovak@fai.utb.cz
}

\section{KEYWORDS}

Predictive control, Local Model Networks, Heatexchanger, Optimization.

\begin{abstract}
The paper deals with the problem of modelling and control using the Local Model Network (LMN). The idea is based on development of multiple local models for the whole operating range of the controlled process. The local models are then smoothly connected using the validity or weighting functions to provide a nonlinear global model of the plant. For saving the computational load, linear model is obtained by interpolating the parameters of local models at each sample instant and then used in Model Predictive Control (MPC) framework to calculate the future behaviour of the process. The supervisory program, based on a nonlinear global model, computes desired values of manipulated variables leading to minimum utility consumption. The approach is verified in a control of model of a heat exchanger.
\end{abstract}

\section{INTRODUCTION}

Many of the processes in the chemical industry exhibit nonlinear behaviour. Their nonlinearities arise from the dynamics in chemical reactions, thermodynamic relationships, etc. Such processes are difficult to model and control. For such problems, there is a strong intuitive appeal in building systems which operate over a wide range of operating conditions by decomposing them into a number of simpler linear modelling or control problems. Multiple-model approaches for the modelling and control of nonlinear systems were proposed in the last decade (Murray-Smith and Johansen, 1997). Here local models are identified over the operating range of the process and form a global nonlinear model process by incorporating the validity function for each of these models (Johansen and Foss 1995).

The basic principles of this modelling approach have been more or less independently developed in different disciplines like neural networks, fuzzy logic, statistics and artificial intelligence with different names such as local model networks (Johansen and Foss 1993), Takagi-Sugeno fuzzy model (Takagi and Sugeno 1985) or neuro-fuzzy model ( Narendra and Parthasarathy 1990;
Zhang and Morris 2002). Similarities and differences between the LMN, radial basis function network (RBFN), fuzzy and Gaussian process model can be found in (Gregorcic and Lightbody 2008).

Model Based Predictive Control (MBPC) or only Predictive Control is one of the control methods which have developed considerably over a few past years. The main advantage of this methodology is that it enables a simple treatment of input and output constraints (Maciejowski 2002), and copes in a natural way with multivariable systems.

There are two ways to design controllers for local model structures, the linearization based and the local model-based approach. For linearization-based approach the local model network is linearized at the current operating point and linear controller is designed. The linearization of the LM network is very simple due to the structure of the model. In the second realisation a local controller is designed for each local model and the control output is then calculated as an interpolation of the local controller according to the current operating point. Both realisations have been widely used in the literature for control of nonlinear systems (Johansen et al., 2000;Mollov et all. 1999).

In this paper the operating regions are distributed in the operating space using the steady-state characteristic of the process. The data for identification of the local models were created by application perturbations around the chosen operating points. The local models were identified using the least-squared method. The parameters of the validity functions were then optimized by minimizing the output error. Thus a nonlinear global model of the process was obtained. Predictive control strategy that uses parameters obtained from linearization of the global model was then applied to provide set point tracking of the output of the plant.

The paper is organized as follows: Section II presents an overview of the local model network approach. The basics of Model Predictive Control are outlined in Section III. In Section IV, the heat-exchanger process is described. Section V describes the modelling of a heatexchanger using the LMN approach. In Sections VI a VII results of predictive control with and without the utility costs taken into consideration are presented. 


\section{LOCAL MODEL NETWORKS}

The basics of Local Model Networks approach are to decompose the operating space into zones where linear models are adequate approximations of dynamic behaviour within that regime, with a trade-off between the number of regimes and the accuracy of the global model. The architecture of LMN benefits from being able to incorporate the a priori knowledge about the system and conventional system identification methodology. The LMN structure also gives transparent and simple representation of the nonlinear system.

In the LMN representation, each model is a local approximation of the modelling surface over a subspace of the operating space, which can be seen as an operating regime (Figure 1).
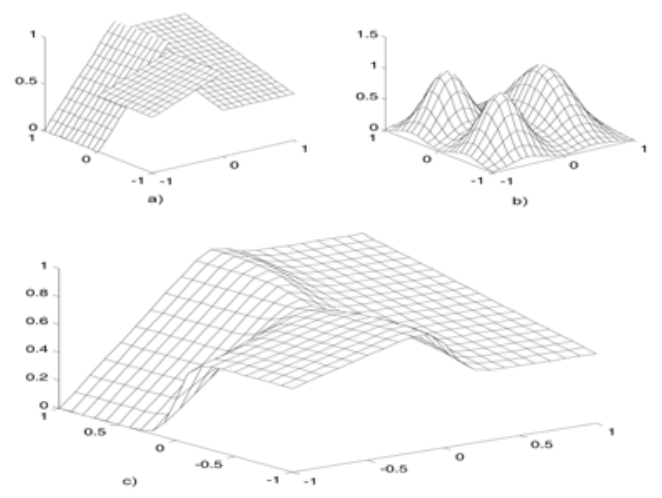

Figure 1: The nonlinear input/output approximation (c) is obtained by combining three linear models (a) with validity functions $(b)$

If linear local models are assumed, the structure of the $i$ th submodel can be defined as follows:

$$
\begin{aligned}
\hat{y}_{i}(k)= & -a_{i 1} y(k-1)-a_{i 2} y(k-2)-\ldots-a_{i n} y(k-n) \\
& +b_{i 1} u(k-1)+b_{i 2} u(k-2)+\ldots+b_{n}^{i} u(k-m) \\
& +d_{i}+\eta(k)
\end{aligned}
$$

where $n$ and $m$ are the numbers of delayed input and output samples, is the $d_{i}$ offset term, and $\eta(k)$ is assumed to be white noise. Eq. 1 can be rewritten to regression form:

$$
\hat{y}_{i}(k)=\boldsymbol{\psi}(k-1)^{T} \boldsymbol{\Theta}_{i}+\eta(k)
$$

where the repressor vector $\psi(k-1)$ is given as

$$
\begin{aligned}
\psi(k-1)= & {[-y(k-1), \ldots,-y(k-n),} \\
& u(k-1), \ldots, u(k-m), 1]
\end{aligned}
$$

and the vector of parameters as:

$$
\Theta_{i}=\left[a_{i 1}, \ldots, a_{i n}, b_{i 1}, \ldots, b_{i m}, d_{i}\right]
$$

The LMN structure can then be represented as follows:

$$
\hat{y}(k+1)=\sum_{i=1}^{M} \varphi_{i}(\phi(k)) \psi(k)^{T} \Theta_{i}
$$

The validity functions $\varphi_{i}(k)$ are functions of some scheduling vector $\phi(k)$. The scheduling vector, which defines the operating point of the system, can include any system state or variable. Although any function with locally limited activation might be applied as a validity function, a common choice is Gaussian or a BSpline function. The Gauss function for $i$-th model is given by

$$
\tilde{\varphi}_{i}(\phi(k))=\exp \left(-\frac{1}{2}\left(\phi-c_{i}\right)^{T} \sigma_{i}^{-2}\left(\phi-c_{i}\right)\right)
$$

The validity functions are generally normalized:

$$
\varphi_{i}(\phi(k))=\frac{\tilde{\varphi}_{i}(\phi(k))}{\sum_{j=1}^{M} \tilde{\varphi}_{j}(\phi(k))}
$$

Normalization of the validity functions is often motivated by the desire to achieve a partition of unity. By partition of unity it is meant that at any point in the input space sum of all normalized validity functions equals unity, i.e.

$$
\sum_{i=1}^{M} \varphi_{i}(\phi)=1
$$

However, normalization also leads to a number of other important side-effects that have consequences for the resulting network (Shorten and Murray-Smith 1997).

Assuming that both the validity functions of the LMN and the parameters of the local model there are known there are two ways of interpolating between the local models:

- A weighted sum of the outputs of local models is used to produce an output of the LMN (Figure 2)

- Assuming all the local models have the same structure parameters of the local models are blended to form an LMN

The blending of parameters provides better transparency, however, cannot be used in applications where the structure of the local models changes across the operating space.

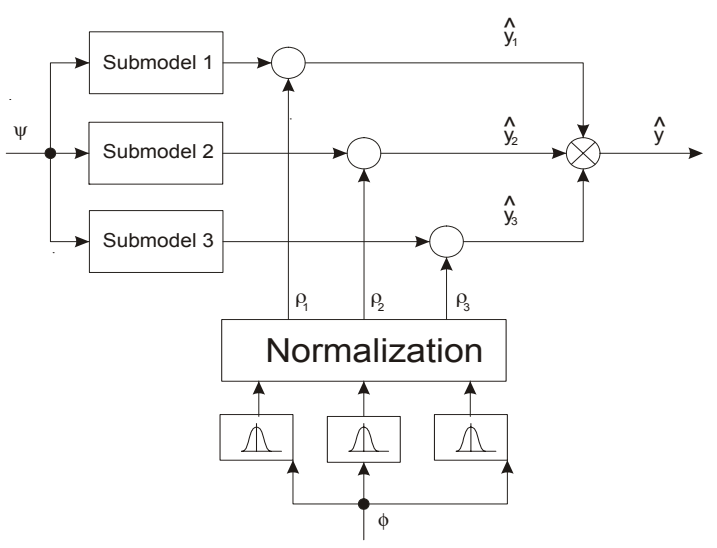

Figure 2: Local model network structure

\section{MODEL PREDICTIVE CONTROL}

In general, industrial processes are nonlinear, but most MPC applications are based on the use of linear models. The first reason is that identification of a linear model is relatively simple and the linear models are sufficient when the plant is operating in the neighbourhood of the operating point. Secondly, the use of linear models with 
quadratic objective function gives rise to a convex problem which can be solved by Quadratic Programming.

If all of the local models share the same structure across the operating space, the parameters of the local models can be blended:

$$
\hat{y}(k+1)=\psi(k)^{T} \sum_{i=1}^{M} \varphi_{i}(\phi(k)) \Theta_{i}+\eta(k)
$$

The structure of the overall model in this case, remains the same as the structure of the individual local models. A predictor in a vector form is then given by

$$
\widehat{\boldsymbol{y}}=\boldsymbol{G} \Delta \boldsymbol{u}+\boldsymbol{Y}
$$

where $\hat{y}$ is a vector of system predictions along the prediction horizon $N, \Delta \mathrm{u}$ is a vector of control increments, $\boldsymbol{Y}$ is the free response vector. $\boldsymbol{G}$ is a dynamics matrix.

The computation of a control law of MPC is based on minimization of the following criterion

$$
\begin{gathered}
J=\sum_{j=1}^{N}\|\hat{y}(k+j \mid k)-w(k+j)\|_{Q}^{2} \\
+\sum_{j=1}^{N}\|\Delta u(k+j-1)\|_{R}^{2}
\end{gathered}
$$

where $\hat{y}(k+j \mid k)$ is a $j$ steps ahead prediction of the system, $w(k+j)$ is a future reference trajectory and $\boldsymbol{Q}, \boldsymbol{R}$ are the positive definite weighting matrices.

The reference trajectory vector

$$
\boldsymbol{w}=[w(k+1), \ldots, w(k+N)]^{T}
$$

is obtained from the first-order lag system:

$$
w(k)=\alpha w(k-1)+(1-\alpha) \omega
$$

where $\alpha$ is an adjustable parameter of the reference trajectory and $\omega$ is the output set point.

The criterion 11 can be rewritten to more general matrix form:

$$
J(k, N)=(\boldsymbol{w}-\hat{\boldsymbol{y}})^{T} \boldsymbol{Q}(\boldsymbol{w}-\hat{\boldsymbol{y}})+\Delta \boldsymbol{u}^{T} \boldsymbol{R} \Delta \boldsymbol{u}
$$

Using the expression (10) it can be simplified

$$
J(k, N)=\Delta \boldsymbol{u}^{T} \boldsymbol{H} \Delta \boldsymbol{u}+2 \boldsymbol{g}^{T} \Delta \boldsymbol{u}+\boldsymbol{f}
$$

where the gradient $\boldsymbol{g}$ and the Hess matrix $\boldsymbol{H}$ are defined by following expressions

$$
\begin{aligned}
& \boldsymbol{H}=\boldsymbol{G}^{T} \boldsymbol{Q} \boldsymbol{G}+\boldsymbol{R} \\
& \boldsymbol{g}=(\boldsymbol{Y}-\boldsymbol{w})^{T} \boldsymbol{Q} \boldsymbol{G} \\
& \boldsymbol{f}=(\boldsymbol{w}-\boldsymbol{Y})^{T} \boldsymbol{Q}(\boldsymbol{w}-\boldsymbol{Y})
\end{aligned}
$$

Since the vector $\boldsymbol{f}$ is a constant vector it does not have an effect on the quadratic programming results. The constraints for the control signal and control signal increments are given:

$$
\boldsymbol{A}=\left[\begin{array}{c}
\boldsymbol{A} \Delta \boldsymbol{u} \leq \boldsymbol{b} \\
I_{u} \\
-I_{u} \\
I_{\Delta u} \\
-I_{\Delta u}
\end{array}\right] \quad \boldsymbol{b}=\left[\begin{array}{c}
u^{\max }-I u(k-1) \\
-u^{\min }+I u(k-1) \\
\Delta u^{\max } \\
-\Delta u^{\min }
\end{array}\right]
$$

Parameters $u^{\max }$ and $u^{\min }$ are constraints for the control signals and $\Delta u^{\max }$ and $\Delta u^{\min }$ are constraints for the control signal increments. Matrices $I_{u}$ and $I_{\Delta u}$ are defined as:

$$
\begin{gathered}
I_{\Delta u}=\left[\begin{array}{cccc}
1 & 0 & \cdots & 0 \\
0 & 1 & 0 & \vdots \\
\vdots & 0 & \ddots & 0 \\
0 & \cdots & 0 & 1
\end{array}\right], \\
I_{u}=\left[\begin{array}{cccc}
1 & 0 & \cdots & 0 \\
1 & 1 & 0 & \vdots \\
\vdots & 1 & \ddots & 0 \\
1 & \cdots & 1 & 1
\end{array}\right] .
\end{gathered}
$$

\section{DESCRIPTION OF THE PROCESS}

Heat exchangers are widely used in space heating, petrochemical plants or power plants. It is represented by a vessel (see Figure 3 ) filled with water. It is possible to increase the water temperature with electric heating A. Heating power $E$ is controlled continuously. Cooling helix $\mathrm{B}$ is used to decrease water temperature $T_{C}$.

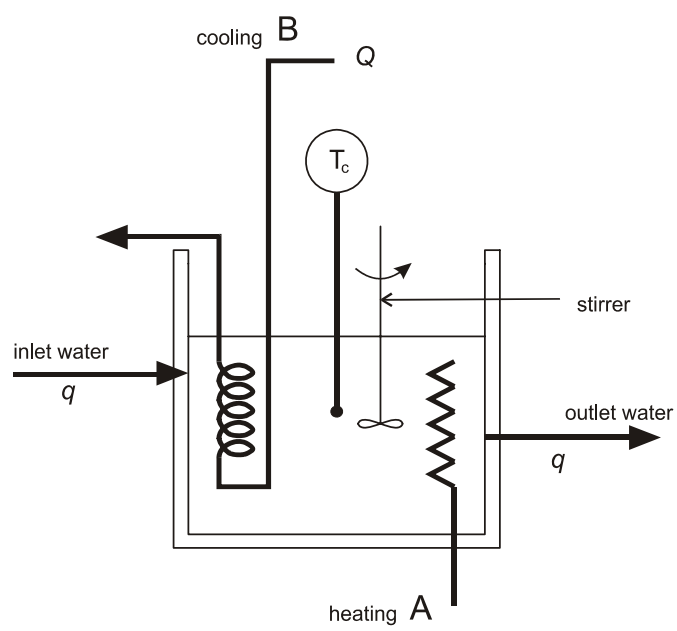

Figure 3 Scheme of a heat-exchanger

The system can be described under a simplified assumption (ideal mixing, characteristic temperatures, etc.) by 3 differential equations (Dusek and Honc 2009).

$$
\begin{aligned}
m_{A} c_{A} \frac{d T_{A}}{d t} & =\alpha_{A} S_{A}\left(T_{c}-T_{A}\right)+E \\
m_{B} c_{B} \frac{d T_{B}}{d t} & =\alpha_{B} S_{B}\left(T_{c}-T_{B}\right)+c_{B} Q\left(T_{B 0}-T_{B}\right) \\
m_{C} c_{C} \frac{d T_{C}}{d t} & =\alpha_{A} S_{A}\left(T_{A}-T_{C}\right)+\alpha_{B} S_{B}\left(T_{B}-T_{C}\right) \\
& +\alpha_{C} S_{C}\left(T_{O}-T_{C}\right)+q c_{C}\left(T_{C 0}-T_{C}\right)
\end{aligned}
$$

where $T_{x}$ are characteristic temperatures

$T_{B 0}$ is input temperature of cooling helix

$T_{0}$ is ambient temperature

$T_{C 0}$ is input temperature 
$m_{x}$ are masses

$c_{x}$ are specific heat capacities

$S_{x}$ are heat transfer areas

$\alpha_{x}$ are heat transfer coefficients.

The input variables are constrained - cooling water flow is within the range $0 \leq Q \leq Q_{\max }$, and heating power $0 \leq E \leq E_{\max }$.

The parameters of the system that are used in the simulations are given in Table 1

Table 1 Parameters of the system

\begin{tabular}{|c|c|c|c|}
\hline Par. & Value & Par. & Value \\
\hline$m_{A}$ & $0.5 \mathrm{~kg}$ & $S_{A}$ & $0.0095 \mathrm{~m}^{2}$ \\
\hline$m_{B}$ & $0.1 \mathrm{~kg}$ & $S_{B}$ & $0.065 \mathrm{~m}^{2}$ \\
\hline$m_{C}$ & $4.0 \mathrm{~kg}$ & $S_{C}$ & $0.24 \mathrm{~m}^{2}$ \\
\hline$c_{A}$ & $452 \mathrm{Jkg}^{-1} \mathrm{~K}^{-1}$ & $\alpha_{A}$ & $750 \mathrm{Jm}^{-2} \mathrm{~s}^{-1} \mathrm{~K}^{-1}$ \\
\hline$c_{B}$ & $4180 \mathrm{Jkg}^{-1} \mathrm{~K}^{-1}$ & $\alpha_{B}$ & $500 \mathrm{Jm}^{-2} \mathrm{~s}^{-1} \mathrm{~K}^{-1}$ \\
\hline$c_{C}$ & $4180 \mathrm{Jkg}^{-1} \mathrm{~K}^{-1}$ & $\alpha_{C}$ & $5 \mathrm{Jm}^{-2} \mathrm{~s}^{-1} \mathrm{~K}^{-1}$ \\
\hline$T_{C 0}$ & $25^{0} \mathrm{C}$ & $q$ & $0.0016 \mathrm{kgs}^{-1}$ \\
\hline$T_{B 0}$ & $15^{0} \mathrm{C}$ & $T_{0}$ & $25^{0} \mathrm{C}$ \\
\hline$E_{\min }$ & $0 \mathrm{kgm}^{2} \mathrm{~s}^{-3}$ & $Q_{\min }$ & $0 \mathrm{kgs}^{-1}$ \\
\hline$E_{\max }$ & $500 \mathrm{kgm}^{2} \mathrm{~s}^{-3}$ & $Q_{\max }$ & $0.0083 \mathrm{kgs}^{-1}$ \\
\hline
\end{tabular}

\section{MODELLING OF HEAT EXCHANGER}

The local modelling approach was used to model the dynamic and steady-state properties of the system. The process exhibits nonlinear behaviour as can be seen from steady-state characteristics depicted in Figure 4.

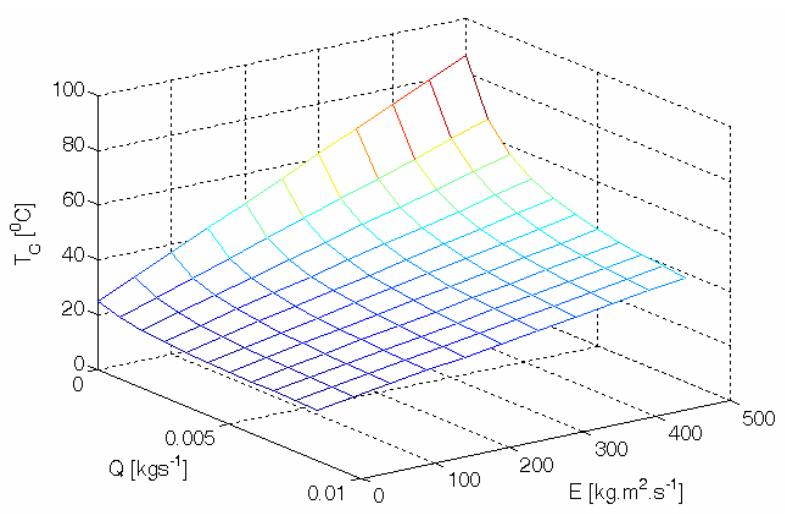

Figure 4. Steady-state characteristics of the heat exchanger

Five separate sets of data were collected at different operating points. The operating points were chosen at the corners of the operating region and in the centre of it. Each data set is restricted to be close to the locus of equilibrium points. To get representative data for each subspace, an additional low-amplitude excitation signal has to be injected into the system. In this way the system is excited locally at the particular operating point and the collected data will reflect the dynamic which is a valid representation of the system only on the subspace close to operating point. Away from those points the accuracy of the network can decrease rapidly due to unmodelled off-equilibrium dynamics.

At each chosen point an affine ARX model

$$
M_{i}: y_{i}(k+1)=b_{1} u_{1}(k)+b_{2} u_{2}(k)+a y(k)+c
$$

was identified. The scheduling vector $\phi(k)$ contains the values of input $u_{1}(k-1)$ and $y(k-1)$. The widths of the Gaussian validity functions $\sigma_{i}^{-2}$ were optimized by minimization of a mean-squared modelling error over the training data using the criterion:

$$
J=\frac{1}{N} \sum_{k=1}^{N}(y(k)-\hat{y}(k))^{2}
$$

It is important to highlight here that in many industrial applications it could be difficult or even unsafe to drive the system rapidly across the operating space and hence global data is often not available. The modelling performance of the LMN is shown in Figure 5 where step changes of both inputs are applied to the system being modelled and LMN.
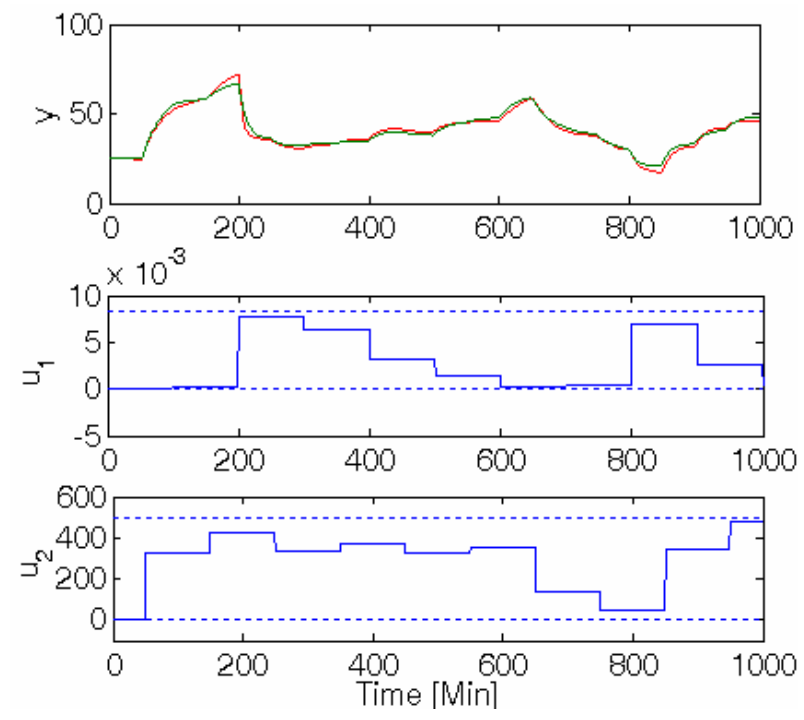

Figure 5. Comparison of the measured output of system (green) and output of the LMN (red) 


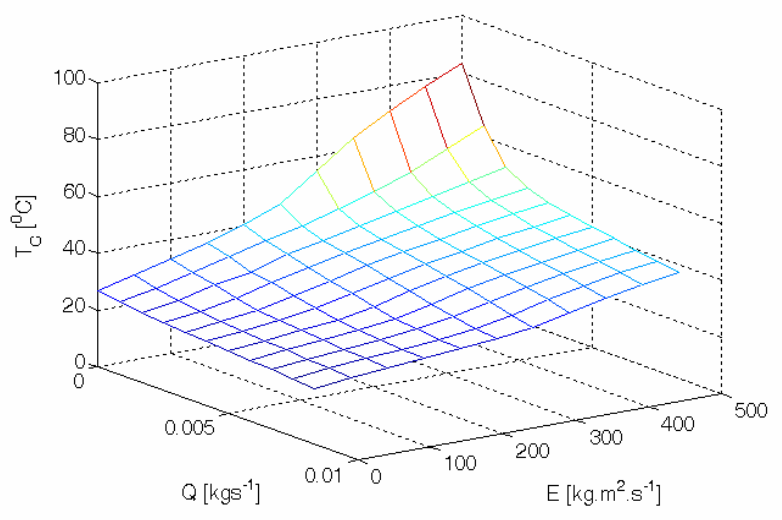

Figure 6. Steady-state characteristics of the LMN

Since the LMN will be used for finding the optimal inputs the steady-state properties of the LMN are of high importance. The steady-state characteristic of the LMN is depicted in Figure 6.

\section{PREDICTIVE CONTROL OF THE HEAT EXCHANGER MODEL}

The control task is to control the temperature inside the vessel $y(k)=T_{c}(k)$ by manipulating the flow-rate of the cooling water $u_{1}(k)=Q(k)$ and heating power $u_{2}(k)=E(k)$. The length of prediction horizon, was set to $N=20$ with sampling period of 1 minute. The weighting matrices $Q, S$ were chosen as

$$
\begin{aligned}
& Q=3 I \\
& S=\left[\begin{array}{cccc}
S_{1} & 0 & \cdots & 0 \\
0 & S_{1} & & 0 \\
\vdots & & \ddots & \vdots \\
0 & 0 & \cdots & S_{1}
\end{array}\right] \\
& S_{1}=\left[\begin{array}{cc}
1000 & 0 \\
0 & 0.1
\end{array}\right]
\end{aligned}
$$

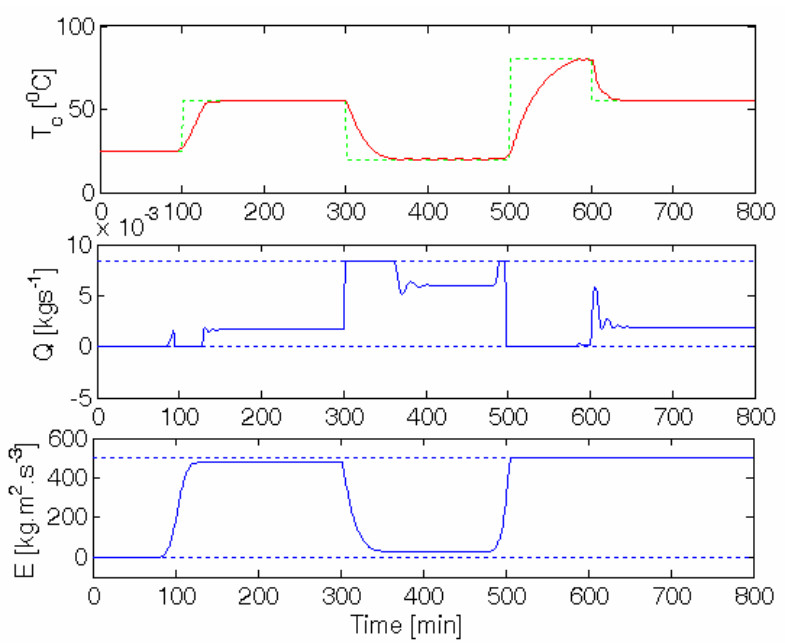

Figure 7 Control of a heat exchanger

Figure 7 shows the dynamic responses of the system when the sequence of changes is introduced into the system. The heating and cooling act against each other. As can be seen at the time from 100 to 300 minutes both inputs are active. The output temperature can be reach with an infinite number of combinations of inputs. However, only one combination of inputs has minimal utility costs.

\section{PREDICTIVE CONTROL WITH MINIMAL COST}

In the MPC strategy an extra term in the cost function of problem (14) is added to account for the utility cost. Thus, the control scheme uses two level structure that consists of supervisory level that determines the optimal steady-state inputs and MPC level that guides the process to this point. The optimization problem of the supervisory level is the minimization of the total utility consumption:

$$
\begin{aligned}
& J\left(u_{1}, u_{2}\right)=c_{1} u_{1}+c_{2} u_{2} \\
& \text { subject to: } \\
& L\left(u_{1}, u_{2}\right)=w(k+N)
\end{aligned}
$$

where $c_{1}, c_{2}$ are the cold and hot utility costs per unit and $L\left(u_{1}, u_{2}\right)$ represents the steady-state response of the system given the inputs $u_{1}, u_{2}$.

The desired optimal values that are obtained at supervisory level are included in the cost function of the control problem as a new term that penalizes the distance that the system is from the desired condition. In (Dusek and Honc 2009) the difference between the optimal state and state at the end of horizon is used. Here the difference between the last control signal and optimal control signal is weighted in the criterion. The MPC optimization problem is reformulated as follows:

$$
\begin{aligned}
J(k, N)= & (\boldsymbol{w}-\hat{\boldsymbol{y}})^{T} \boldsymbol{Q}(\boldsymbol{w}-\hat{\boldsymbol{y}})+\Delta \boldsymbol{u}^{T} \boldsymbol{R} \Delta \boldsymbol{u} \\
& +(\boldsymbol{K} \Delta \boldsymbol{u}-\boldsymbol{l})^{T} \boldsymbol{S}(\boldsymbol{K} \Delta \boldsymbol{u}-\boldsymbol{l})
\end{aligned}
$$

where matrix $\boldsymbol{K}$ and vector $\boldsymbol{l}$ are defined as:

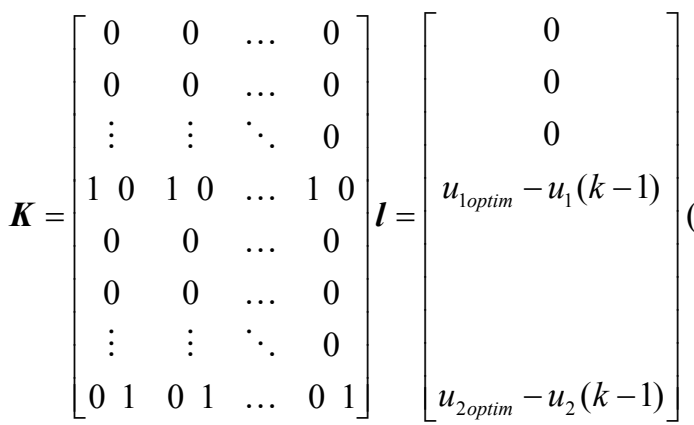

Only the future final value is used in the criterion to separate the effect of the optimising action of the supervisory level from the more immediate MPC closed-loop corrections. Analysis of this technique concerning the model plant mismatch can be found in (Gonzales et al. 2006). The main control objective is to follow the set-point and respect manipulated variable constrains and at the same time to minimize energy costs for heating and cooling. The same horizons and weighting matrices as in the previous control have been 
used in the criterion. Additional weighting matrix $\mathrm{S}$ was chosen as

$$
\begin{aligned}
& S=\left[\begin{array}{cccc}
S_{1} & 0 & \cdots & 0 \\
0 & S_{1} & & 0 \\
\vdots & & \ddots & \vdots \\
0 & 0 & \cdots & S_{1}
\end{array}\right] \\
& R_{1}=\left[\begin{array}{cc}
1000 & 0 \\
0 & 0.1
\end{array}\right]
\end{aligned}
$$

The prices utilities at the supervisory level control were set to $c_{1}=0.01$ and $c_{2}=1$ to reflect that prices for heating are much higher than prices for cooling. At each step change of reference value the optimal values of inputs with minimal utility costs are computed.
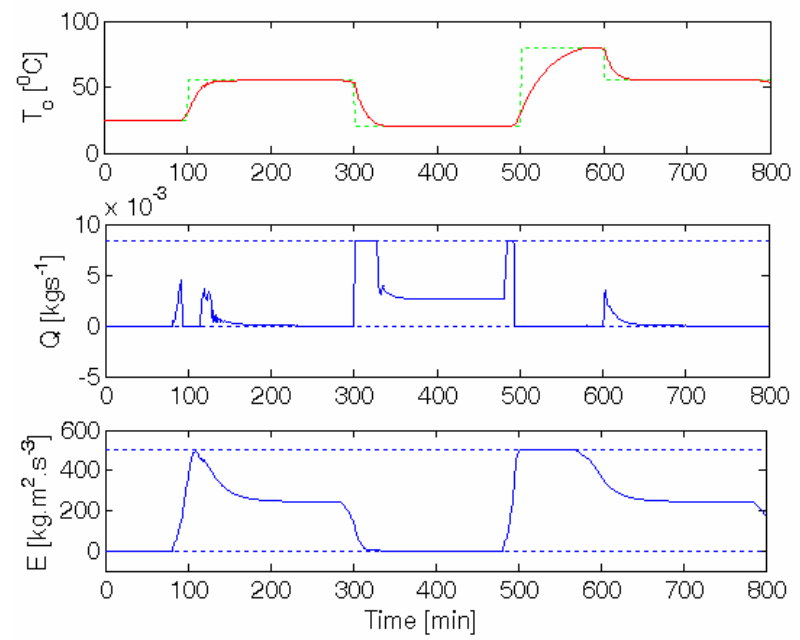

Figure 8 Control of a heat exchanger with minimal costs

As can be seen, now the cooling is used only for decreasing the temperature or if the setpoint temperature that is less then ambient temperature. The optimal input conditions are calculated based on an approximated non-linear model. The closer the non-linear model utilized in the steady-state optimization is from the process, the more consistent will be the targets and set points sent to the control system.

Despite of steady-state model mismatch, by penalizing only the last control action in the control horizon, the MPC remains free to use the remaining control actions to satisfy dynamic requirements and set point targets. The final response to the system with supervisory control for minimal utility costs is depicted in Figure 8.

\section{CONCLUSION}

In this paper, a MPC scheme based on local model network has been applied to model of a heat exchanger. The operation of the process is decomposed into a set of operating regimes, and simple local models are developed for each regime. These are combined into a global model structure using the validity functions. The supervisory level looks for steady-state input values in agreement with minimum utility consumption, while the MPC accounts for transient performance and offset free controlled outputs.

\section{ACKNOWLEDGMENT}

The work has been supported by Ministry of Education of the Czech Republic under the grant 102/09/P243.

\section{REFERENCES}

Dušek F., D. Honc. 2009. "Control of a simple constrained MIMO system with steady state optimization - case study". In Proceedings of 28th IASTED International Conference on Modelling, Identification and Control, Innsbruck, Austria.

Gonzales A.H., D. Odloak and J. L. Marchetti. 2006. "Predictive control applied to heat-exchanger networks". Chemical Engineering and Processing. Vol. 45 (Aug), 661-671.

Gregorčič G. and G. Lightbody. 2008. "Nonlinear system identification: From multiple-model networks to Gaussian processes". Engineering Applications of Artificial Intelligence, vol. 21 (Oct), 1035-1055.

Johansen, T.A., Foss, B.A., 1992. "A NARMAX model representation for adaptive control based on local models". Modeling Identification and Control 13, 25-39.

Johansen T.A. and B.A. Foss. 1995. "Identification of nonlinear system structure and parameters using regime decomposition". Automatica, Vol. 31, 321-326.

Johansen, T.A., K. Hunt and I. Petersen. 2000. "Gain scheduled control of a solar power plant". Control Engineering practice, Vol. 8, 1011-1022.

Maciejowski J.M. 2002. Predictive Control with Constraints. Prentice-Hall. Harlow, England.

Mollov S., P. Veen, R. Babuska, J. Abonyi, J. Roubos and H.B. Verbruggen. 1999. "Extraction of local linear models from Takagi-Sugeno fuzzy model with application to model-based predictive control. In Proceedings of $7 \mathrm{th}$ European Congress on Intelligent Techniques and Soft Computing. Aachen, Germany.

Murray-Smith R. and T.A. Johansen. 1997. Multiple Model Approaches to Modelling and Control. Taylor and Francis, London.

Narendra K.S. and K. Parthasarathy. 1990. "Identification and control of dynamical systems using neural networks". IEEE Transactions on Neural Networks, 4-27.

Shorten R. and R. Murray-Smith. 1996. "Side effects of Normalising Radial Basis Function networks". International Journal of Neural Systems. Vol. 7.

Takagi, T. and Sugeno, M. 1985. "Fuzzy identification of systems and its applications to modeling and control". IEEE Transactions on Systems, Man and Cybernetics, 116-132.

Zhang, J. and A.J. Morris. 2002. "Long Range Predictive Control of Nonlinear Processes Based on Recurrent Neuro-Fuzzy Network Models". Neural Computing and Applications. Vol. 9.

\section{AUTHOR BIOGRAPHIES}

JAKUB NOVAK was born in Zlin, Czech Republic and received the Ph.D. degree from the Tomas Bata University, Zlin, Czech Republic in 2007. He is now a researcher at the Center for Applied Cybernetics at Tomas Bata University. His research interests are multiple model strategies and predictive control. His email address is : jnovakefai.utb.cz 